\title{
EL ALUMNO SOBRESALIENTE, SU EDUCACIÓN Y LA ATENCIÓN A SUS NECESIDADES ESPECIALES EN EL SISTEMA EDUCATIVO COSTARRICENSE
}

\author{
Giuseppa D’Agostino Santoro
}

\author{
Recibido 10-V-2003 • Aceptado 10-VI-2003
}

\begin{abstract}
Resumen: Las páginas aquí presentadas constituyen una reflexión analítica-descriptiva acerca de la educación del aprendiz sobresaliente y talentoso en el área académica. Para ello, primero se efectúa una somera caracterización de este tipo de alumno y se brinda una visión de las alternativas educacionales más aplicadas en la práctica pedagógica, para su formación. Seguidamente, se aportan algunas acciones estatales, efectuadas a nivel de política del sistema educativo costarricense para atender las necesidades de este colectivo de alumnos y se evidencia su situación actual de descuido en dicho sistema. Se concluye evidenciando la presencia de una visión reduccionista de las necesidades educativas especiales en la Normativa pertinente, lo cual contraviene la filosofía de la educación inclusiva, y el derecho a la diversidad y a una educación diversificada de todos y cada uno de los alumnos.
\end{abstract}

Palabras clave: Alumno Sobresaliente, Alternativas de Formación, Políticas Educativas, Diversidad y Necesidades Educativas Especiales (NEE).
El grado de productividad cultural y el continuo avance científico y tecnológico que caracterizan nuestra época requieren el aporte del talento, de la inteligencia e ingeniosidad que poseen los individuos altamente dotados. En gran medida, son sus contribuciones las que permiten ampliar los horizontes del conocimiento, solucionar los problemas que apremian la sociedad e incentivar el progreso en los distintos campos de la actividad humana.

De acuerdo con esta visión, es irracional para el mejoramiento y la evolución del ser humano descuidar, tal como se ha hecho en el pasado y todavía se continúa haciendo en algunos países, incluido el nuestro, la identificación precoz y la educación de los niños que poseen capacidad intelectual y desempeño superior. Ningún tipo de sociedad debe y puede correr el riesgo de perder y desaprovechar un potencial tan valioso para su desarrollo ético, artístico, literario, político, económico y demás.

La idea equívoca de que el niño bien dotado puede abrirse camino por sí mismo sin una ayuda específica que le provea oportunidades aptas para el desarrollo de su potencial y talento, ha constituido una rémora para su detección y para el surgir y la puesta en práctica de experiencias pedagógicas diversificadas. Es factible que aún sin proporcionarle ayuda y servicios especiales, algunos niños dotados logren 
un desempeño notable; pero ello no significa, en absoluto, el avance del desarrollo máximo de sus capacidades, de su autonomía y de su bienestar emocional y social.

Dentro de una sociedad democrática, el alumno talentoso, lo mismo que cualquier otro, tiene el derecho a un tratamiento educacional que abarque desde el período preescolar hasta los niveles educativos más altos y que, además, favorezca el desarrollo completo de su potencial, de su autorrealización y de sus posibilidades de hacer contribuciones significativas para el cambio y el mejoramiento de la sociedad y de la vida humana.

Este sentido de justicia y de igualdad de oportunidades, junto a las exigencias propias de la complejidad cultural y social de nuestra sociedad, aunado a la investigación científica acerca de las personas dotadas, han contribuido al aumento progresivo del interés que, hoy en día se registra en múltiples países hacia la identificación y la educación de los individuos talentosos. Si bien la realización de estas tareas varía de país en país, en términos generales, se constatan esfuerzos y adelantos ya sea en los sistemas de detección como en el planeamiento de programas y servicios educativos oportunos.

Es nuestro interés exponer, enseguida, estos programas de formación y servicios educativos, pero no sin antes ofrecer al lector una somera conceptualización del término alumno sobresaliente y talentoso.

\section{Significado del término alumno sobresaliente y talentoso}

Es amplia y variada la terminología que en el pasado ha sido utilizada y que se emplea aún para identificar al alumno sobresaliente. Se le ha llamado genio, dotado, brillante, talentoso, precoz, superdotado, superior, prodigio y muchos otros. Sin entrar en los detalles específicos de estas opciones terminológicas, es evidente que, en general, apuntan a que el alumno sobresaliente es el que posee habilidades y dotes superiores a la mayoría de sus coetáneos. Se trata de capacidades extraordinarias para el medio socio-cultural en el que está inmerso y de inusitadas aptitudes y características intelectuales, de personalidad, sociales, psicológicas y aún físicas que constituyen su bagaje potencial y que se manifiestan, en muchos casos, desde temprana edad.

Si bien reconocemos que la terminología y la categorización relacionadas con el concepto de talento es rica y varía de una época a otra y de un país a otro, en este contexto, optamos por seleccionar la conceptualización del Departamento de Educación de los Estados Unidos, que refleja un enfoque alejado de la orientación piscométrica del pasado y encierra la visión actual sobre el tema:

"Los niños y adolescentes superdotados muestran respuestas notablemente elevadas, o el potencial necesario para alcanzarlas, comparados con los demás individuos de su misma edad, experiencia o entorno. Poseen altos niveles de capacidad en las áreas cognitivas, creativas y/o artísticas, demuestran una capacidad excepcional de liderazgo o destacan en asignaturas académicas específicas. Estos alumnos necesitan servicios y actividades que la escuela ordinaria no suele ofrecer. Las capacidades superiores se dan en niños y adolescentes de todos los grupos culturales, en todos los estratos sociales y en todos los campos de la actividad humana" (Howell y otros 1998, pág. 438).

Esta definición, por su amplitud y diversificación, nos ofrece un panorama multidimensional de las diferentes habilidades y facultades del niño y del adolescente sobresaliente, que pueden evidenciarse de manera aislada o combinada en una o más áreas de la actividad humana. Asimismo, resalta la importancia de las oportunidades del entorno para el desarrollo de las capacidades referidas y la necesidad de identificarlas y atenderlas también dentro de los grupos culturales minoritarios y los estratos económicos en desventaja.

En la actualidad, entonces, se considera como superdotado y talentoso no 
solamente al individuo que posee un alto cociente intelectual (único parámetro utilizado hace unas décadas), sino además, a las personas que demuestran un talento excepcional para el liderazgo, las artes y la música, como también aquellas que tienen creatividad y facultades específicas para distintas disciplinas académicas.

Salta a la vista que el concepto de individuo sobredotado es amplio y debe entenderse en términos de una constelación heterogénea de áreas en las cuales la persona puede presentar potencialidades extraordinarias y un excepcional desempeño. Conscientes de esta diversidad existente dentro de los sujetos altamente dotados, nosotros optamos por ceñirnos dentro del presente contexto al alumno sobresaliente y de notable desempeño en las áreas académicas. Aún con esta restricción, no es fácil delimitar los rasgos que caracterizan a este aprendiz, pues cada persona es única y como tal presenta su propia caracterización psicológica, sociocultural y, motivaciones y habilidades específicas. Así, por ejemplo, un niño sobresaliente de un medio socio-cultural puede presentar rasgos distintos a otro que proviene de un medio diferente. De hecho, la población superdotada y talentosa, al igual que todo grupo humano, no es homogénea, por el contrario, exhibe atributos variados y diferencias individuales considerables. Hecha tal salvedad, encontramos que los alumnos que evidencian altas cualidades en el área intelectual y académica son aquellos niños que tienen en común, una habilidad inusitada para aprender bien y en forma rápida. Son curiosos, capaces de alta concentración y perseverancia en las actividades que les interesan y manifiestan un pensamiento altamente crítico, abstracto, fluido y creativo. Muchos de ellos poseen también, una memoria visual y auditiva extraordinaria, un vocabulario rico y una gran capacidad para solucionar problemas y aplicar conceptos abstractos en circunstancias y situaciones diversas.

En fin, se trata de niños que destacan en su rendimiento escolar global y en áreas académicas específicas, por su alta capacidad intelectual, por su creatividad y por su interés y extraordinaria dedicación a las actividades y experiencias de aprendizaje involucradas en estas áreas. Sin embargo, el hecho de que un ser humano posea en amplia dimensión y profundidad las capacidades aludidas, no significa que las desarrolle, directamente en forma adecuada. Hay múltiples factores que deciden si un niño talentoso logra o no concretar su potencial en algo valioso y creativo para él y la humanidad. Entre esos factores destacan la calidad de su medio familiar y escolar, la orientación, el estímulo que recibirá a lo largo de su desarrollo, el clima de aceptación con el que contará y las oportunidades culturales y educativas que dispondrá. Son estos factores que entre otros y en larga medida, determinarán el desarrollo de sus dotes y de sus posibilidades para hacer valiosas contribuciones al bienestar del género humano.

Tales razones justifican una educación diferenciada de los niños dotados. No es suficiente con que estos niños salgan airosos de los cursos regulares que llevan al ritmo de los demás coetáneos; por el contrario, se requieren para ellos oportunidades educativas desafiantes que potencien sus talentos y destrezas y que favorezcan el pleno desarrollo de su personalidad. En el ambiente académico, es bien conocido que el prescindir de un tratamiento educativo diferenciado, apto para desarrollar las facultades de estos alumnos, conlleva a su fracaso escolar y personal, por cuanto la práctica educativa "regular" carece de interés para ellos. Es a estas oportunidades educativas y a su caracterización que dedicaremos los puntos siguientes del presente escrito.

\section{Alternativas educacionales}

La concepción y el desarrollo de opciones educativas y de orientación apropiadas para la formación de los alumnos con talento están vinculadas con el proceso de 
identificación de estos. El propósito principal de tal proceso estriba en detectar al niño sobresaliente a la edad más temprana posible y evaluar sus dotes y condiciones personales. Todo ello, con la finalidad de proporcionarle alternativas y programas educativos y de orientación que respondan a sus necesidades intelectivas, académicas, sociales y emocionales. De tal manera que un óptimo proceso de identificación, además de descubrir al alumno talentoso, debe proporcionar un cuadro claro y real de su desempeño, de sus habilidades cognoscitivas y creativas, actitudes y necesidades. Este perfil del estudiante es la base para cualquier intento de brindar medidas de educación sistemáticas que, además de ser contextualizadas, deben, entonces, necesariamente, ensamblarse con los resultados arrojados por el proceso de identificación y de diagnóstico pedagógico de las potencialidades y necesidades educativas de cada aprendiz sobresaliente. Hecha tal salvedad, caracterizamos las estrategias educacionales que adoptan, en línea general, los países que se interesan por la educación especial de estos aprendices, proporcionando al mismo tiempo, algunas aportaciones para extraer una reflexión personal sobre las oportunidades reales que este colectivo de alumnos dispone, en el sistema escolar costarricense, para efectuar un proceso de aprendizaje significativo y retador.

Entre las estrategias de formación antes aludidas y a las cuales haremos referencia en el punto siguiente, sobresalen la aceleración, el enriquecimiento del currículo y las actividades extracurriculares dentro del marco de los estudios ordinarios y, los programas especiales en clases y grupos homogéneos.

\subsection{Aceleración}

Este tipo de intervención educativa implica toda estrategia que permita al alumno talentoso un avance más rápido en sus estudios. Tal avance se asocia tanto con la ubicación del estudiante en grados, ciclos y modalidades de estudios superiores a los que le corresponden por su edad cronológica, cuanto con la oportunidad de accesar programas educativos de mayor complejidad y envergadura.

Dentro de esta modalidad se destacan prácticas tales como: el ingreso precoz, antes de cumplir la edad cronológica requerida, a la educación preescolar, primaria, secundaria y universitaria; el salto de uno o más grados escolares; el acceso a cursos superiores en las áreas específicas del talento del alumno; la realización de un currículo condensado (por ejemplo, cubrir el programa de dos años en uno) y la acreditación de cursos y años escolares mediante pruebas por suficiencia. Pero, por encima de la estrategia específica que se aplica, la aceleración siempre hace énfasis en el aumento del ritmo del proceso educacional, de tal manera que el aprendiz pueda realizar sus estudios en tiempos más breves de los acostumbrados dentro de la práctica pedagógica regular.

En el ámbito educativo, con frecuencia, se insinúan dos tipos de preocupaciones con respecto a la aplicación de esta modalidad. La primera hace referencia a los posibles problemas de ajuste que el alumno talentoso pueda experimentar al tener que compartir y competir con compañeros de mayor edad cronológica y mayor desarrollo físico y social.

La segunda apela a la posibilidad de que al acortar el tiempo de estudio se comprometan experiencias y habilidades básicas en el desarrollo del estudiante. En la realidad, los estudios investigativos efectuados demuestran que si la aceleración se hace de manera correcta conlleva pocos o ningún problema. Sobre esta línea, Howell y otros citan la posición de Feldhusen (gran defensor de la aceleración como servicio educativo) con respecto al proceso contrario, que reproducimos enseguida:

"Una importante pregunta que debemos plantear es qué sucede si no se adelanta a los alumnos. El insoportable 
aburrimiento diario (Feldhusen y Kolloff, 1985), los problemas de comunicación con compañeros menos maduros intelectualmente, y las bajas expectativas sobre los contenidos educativos pueden provocar graves problemas emocionales a estos niños. Algunos alumnos con capacidades muy altas pueden sufrir un verdadero ostracismo a causa de sus avanzados intereses y su precoz conducta verbal (...) Así, hay muchas razones para pensar que los problemas de la adaptación social y/o emocional de los alumnos superdotados pueden ser mayores si se les integra según su edad que en cursos avanzados." (Howel y otros 1998, pág. 467).

Compartimos la preocupación señalada por Feldhusen y destacamos que aplicar un programa de aceleración es, siempre, mejor que no hacer nada. Sus posibles inconvenientes se obvian si se realiza como un verdadero plan educativo; diseñado, puesto en práctica y monitoriado, para atender de manera apropiada las necesidades de aprendizaje y el desarrollo del alumno particular al cual está dirigido. Evidentemente en esta, como en cualquiera otra modalidad que se adopte, para un óptimo resultado se requieren condiciones precisas antes, durante y al término del proceso, tales como una buena valoración de las habilidades, de los intereses, de la motivación y adaptación del alumno dentro del entorno en el cual se desenvuelve, el apoyo y la orientación psicopedagógica oportuna de parte del profesor o de los profesionales a cargo del plan educacional que se aplica y, la escogencia de los itinerarios y ambientes formativos que mejor respondan a la situación específica del caso de interés.

\subsection{Enriquecimiento curricular y extracurricular}

El enriquecimiento del currículo en el aula común consiste en ofrecer al alumno sobresaliente alternativas educacionales que amplíen, profundicen y enriquezcan el currículo estándar, de manera tal que este concuerde más con sus intereses y necesidades. El aprendiz talentoso, amen de sus diferencias individuales contextualizadas, requiere para el desarrollo de sus potencialidades, propuestas formativas intelectualmente estimulantes, desafiantes y libres de aspectos rutinarios, repetitivos y aburridos. No se trata, entonces, solo de modificar la propuesta curricular incrementando la cantidad de tareas y de contenidos objeto de estudio, sino de privilegiar ambientes de aprendizaje y experiencias formativas que potencien las habilidades de pensamiento y permitan al estudiante interactuar, experimentar e innovar con el contenido conceptual.

En tal sentido Howell y otros hacen referencia a los elementos esenciales que, según Maker (1982), pueden ser objeto de cambios dentro de un programa de enriquecimiento curricular para alumnos talentosos. Estos elementos ejes son constituidos por el contenido, el proceso de formación, el producto educativo y el entorno. $\mathrm{Al}$ respecto así se manifiestan los autores antes mencionados:

“1 Modificaciones del contenido. Los contenidos curriculares son las asignaturas que se enseñan. En general, el objetivo consiste en desarrollar contenidos más avanzados, complejos, innovadores y originales que los que se presentan a los alumnos en el aula ordinaria.

1 Modificaciones del proceso educativo. Las estrategias y métodos que se aplican para enseñar los contenidos a los alumnos son un aspecto básico de la educación. El objetivo consiste en proporcionar a los alumnos numerosas oportunidades para responder frecuentemente a los contenidos curriculares, lo que incluye las investigaciones independientes, el aprendizaje cooperativo, la ayuda de los compañeros, las simulaciones y las prácticas.

1 Modificaciones de los productos educativos. Los productos educativos son los resultados del aprendizaje. El objetivo consiste en elaborar diversas maneras en que los alumnos puedan mostrar los resultados de su aprendizaje.

1 Modificaciones del entorno. El entorno educativo incluye tanto las características físicas del lugar cuanto el ambiente que crean los profesores y los asistentes educativos. La meta consiste, ante todo, en establecer un entorno positivo de trabajo y luego reordenar 
su diseño. Métodos como la tutorización entre iguales, los centros de interés, las horas de educación y las unidades didácticas pueden ayudar a los alumnos a controlar más activamente su aprendizaje y a interesarse en él." (Howell y otros 1998, p. 476).

La postura anterior deja en claro que el enriquecimiento curricular es una estrategia de intervención educativa multifacética que implica cambios, tanto en los contenidos curriculares, como en las experiencias de aprendizaje, en las prácticas evaluativas y en las habilidades y destrezas que se potencian. Todo ello insertado y dinamizado en un entorno de aula, motivador, estimulante y creativo. En ocasiones, este tipo de intervención se complementa con actividades extracurriculares (giras, visitas, viajes, clubes de literatura, de ciencias naturales, de matemática, de idiomas, de informática, arte y otros) que el estudiante talentoso puede realizar individualmente o en pequeños grupos, fuera del horario escolar y durante el período vacacional. Esto favorece tanto la formación integral del aprendiz como el desarrollo del mismo currículo regular, porque permite abordar temas y áreas no accesibles dentro del tiempo y espacio del aula ordinaria y que son de especial interés y atractivo para el estudiante involucrado (Alonso J. A. y Benito Y. 1996).

Sin lugar a duda, la estrategia educativa de enriquecimiento constituye una adaptación significativa del currículo ordinario a las necesidades educativas y a los intereses individuales del aprendiz sobresaliente, para lograr el más pleno desarrollo de sus capacidades y potencialidades. Es una respuesta a la diversidad dentro del aula regular en total consonancia con el enfoque pedagógico actual de la Educación Inclusiva (Stainback S. y W. 1999), que hace hincapié en la valorización de la diversidad y en el reto de convertir las escuelas en comunidades educativas que lejos de excluir a alguien, faciliten y promuevan el aprendizaje de todos y cada uno de los alumnos, en el contexto del aula ordinaria. Los planteamientos de este enfoque educacional son de vanguardia y están destinados a proyecciones futuras de amplia envergadura. Ello, por cuanto, tal como lo afirman Perpoint J. y Forest, M.:

“... la inclusión es más que un método, una filosofía o un programa de investigación. Es una forma de vivir. Tiene que ver con el vivir juntos con la acogida al extraño y con volver a ser todos uno. ...La inclusión es un juicio de valor. ...Los educadores tenemos que reclamar y luchar por un sistema público de educación que proporcione una educación de calidad y la igualdad para todos". (Stainback S. y V. 1999. Prólogo).

En consonancia con lo anterior, los sistemas educativos tienen que hacer los cambios pertinentes para responder de manera satisfactoria a las necesidades de todos y cada uno de los aprendices.

"Todos los alumnos tienen derecho a asistir a la escuela ordinaria, en su barrio con los niños de su edad, tengan o no deficiencias físicas, sensoriales o mentales, presenten comportamiento autístico o sean superdotados o presenten problemas de la conducta y dificultades de aprendizaje. La responsabilidad pasa ahora a la escuela, que ha de ser tan flexible, tan enriquecida en medios personales y materiales, que pueda acoger al mayor número de niños permaneciendo en los centros especiales un pequeño colectivo”. (Ortiz M. del C., 2000, p. 6).

Es evidente la envergadura de este nuevo enfoque educativo en la tarea de optimizar los procesos de aprendizaje que se desarrollan en el ambiente del aula ordinaria y de ofrecer un currículo significativo que permita a todos los estudiantes experiencias educativas que tengan sentido y valía para cada uno de ellos. Dentro de esta perspectiva, el enriquecimiento curricular se posiciona como una respuesta conveniente para la educación de los niños sobresalientes en el ámbito heterogéneo del aula ordinaria. Tal medida puede aparentar ser sencilla y fácil de aplicarse, pero en la realidad no es así, pues su éxito constituye todo un reto para la mayoría de los sistemas educativos actuales, por estar estrechamente vinculado con condiciones 
que no siempre son reencontrables en dichos sistemas. Dentro de estas condiciones sobresalen las siguientes:

$1 \quad$ Educación centrada en el estudiante.

$1 \quad$ Flexibilidad del sistema educativo.

1 Preparación, idoneidad y compromiso de los docentes involucrados en el programa.

1 Planeamiento y realización de la experiencia educativa como un verdadero programa de estudio (opuesto a actividades vagas, esporádicas e inconexas), adaptado a los intereses y a las necesidades del alumno del que se trate.

1 Grupos pequeños.

$1 \quad$ Metodología activa y participativa con énfasis en el trabajo investigativo, creativo y retador.

1 Recursos didácticos y entorno de aula tipo taller y laboratorio.

Son estas condiciones las que, en gran medida, harán la diferencia en la posibilidad de éxito de un programa de adaptación del currículo ordinario para aprendices talentosos dentro del contexto del aula regular. De lo contrario este ámbito académico "inclusivo" constituirá para el alumno sobresaliente el ambiente educativo menos apropiado y más restringido para desarrollar sus potencialidades y educarse con el ritmo, la profundidad y la rigurosidad académica que requiere.

\subsection{Programas y agrupamientos especiales}

Esta alternativa de formación consiste en educar al aprendiz talentoso con compañeros que poseen habilidades, intereses, y capacidades similares. Son grupos homogéneos de alumnos, a cargo de profesionales especialmente capacitados para facilitar su formación y la puesta en práctica de programas educativos, previstos y diseñados en función de sus características y necesidades específicas. Todo ello, para favorecer su desarrollo pleno y la profundización de las áreas específicas de su talento. En general son programas instructivos especiales, "que intentan reducir el rango de diferencias individuales y facilitan unas experiencias de aprendizaje muy intensas, amplias o aceleradas, cuya naturaleza difiere sustancialmente de las experiencias escolares regulares" (Porto, A. M., 2000, 86).

Las modalidades del agrupamiento son variadas, se extienden desde la versión más extrema cual es la escuela especial hasta las clases especiales a tiempo total o parcial y la formación de grupos específicos que funcionan por algunas horas todos los días o por días alternos. En cualquiera de estas alternativas, la presencia del grupo homogéneo propicia un ambiente más competitivo y retador del que se puede lograr dentro del grupo heterogéneo del aula regular, lo cual ejerce un efecto beneficioso sobre la motivación del aprendiz y sobre su éxito personal y académico.

"La agrupación homogénea intenta reconocer a cada uno el derecho democrático a una educación adecuada y apropiada a sus peculiaridades, a una educación que le sirve para la obtención de su autorrealización, felicidad y desarrollo pleno en todas las áreas. La agrupación homogénea no implica que los niños sean idénticos, sino que son semejantes" (Alonso J. A. y Benito Mate J. 1992, pág. 226).

No obstante los aspectos positivos antes señalados, esta estrategia pedagógica es objeto de críticas y controversias (Roselló, C. y González, J. P. 1993). En particular, se le tacha de ser una medida altamente costosa, segregacionista y elitista. Sin ningún afán de polemizar en este contexto, sobre la autenticidad de tales argumentos, nosotros partimos de la idea clave de que la atención a la diversidad es un derecho y un 
valor en si mismo, que, en educación, se traduce en proporcionar a cada alumno las oportunidades, las experiencias formativas y los entornos de aprendizaje que mejor satisfacen sus necesidades e intereses. En este sentido, si la agrupación selectiva de los aprendices sobresalientes en clases o grupos especiales representa, en casos específicos, la mejor respuesta educativa, no debería de haber mayores razones para desestimarla como tal.

Para concluir con el perfil aquí trazado sobre estrategias de intervención educativas para el aprendiz talentoso, evidenciamos que las alternativas delineadas no son incompatibles entre sí, por el contrario; dentro de una perspectiva ecléctica (Roselló, C. C. y González, J. P. 1993), es posible integrarlas y conformar propuestas educacionales efectivas y multifacéticas. A manera de ejemplo, se puede atender al alumno talentoso dentro del aula regular con un programa individual de enriquecimiento del currículo y permitirle, al mismo tiempo, participar dos o más horas, durante el horario de clase ordinaria o fuera de él, en un grupo homogéneo con un programa especial en las áreas específicas de su talento. A esto, se suman los amplios aportes del uso de las nuevas tecnologías de la comunicación como recurso formativo para estimular e impulsar, dentro y fuera del ámbito escolar, el desarrollo de las habilidades y estrategias de aprendizaje del alumno y su éxito y avance en el campo particular de su talento.

En fin, el alumno sobresaliente -igual que todos los demás aprendicestiene el derecho de disponer de una gama de itinerarios educativos de los cuales, a partir de su contexto, circunstancias y características individuales, se selecciona la alternativa o las alternativas más favorables para su desarrollo y formación. De aquí, el reto para los sistemas educativos actuales, de emprender políticas y acciones educacionales que den respuestas reales y eficaces a la diversidad.

\section{La atención de las necesidades educativas del alumno sobresaliente en las escuelas del sistema educativo costarricense}

A partir de la mitad de los años setenta en Costa Rica surgen inquietudes con respecto a la urgencia de identificar y atender aquellos estudiantes con talentos especiales que, en la mayoría de los casos, pasan ignorados por las aulas de las escuelas del sistema educativo costarricense, sin oportunidades de experiencias de formación ajustadas a sus necesidades especiales. En el año 1982, el Centro Multinacional de Investigación Educativa de la OEA (CEMIE), el Instituto Educativo Moderno y el Ministerio de Educación Pública (MEP) efectúan un estudio investigativo sobre detección y características del alumno talentoso en Costa Rica (Lafourcade P. y otros, 1982). A raíz de este estudio, el Instituto Educativo Moderno realiza, a lo largo de tres semestres, con un grupo experimental y otro de control, una experiencia educativa de enriquecimiento y flexibilidad curricular dentro del salón de clase regular. Esta experiencia confirma la hipótesis de las investigadoras (Beirute, L. y De Bravo, O. 1984) de que el aprendiz talentoso eleva su índice de creatividad cuando se le dan las oportunidades educativas de un programa curricular enriquecido y se aplica una metodología activa y atención personalizada.

Con base en los resultados de estos dos valiosos estudios, los investigadores involucrados, elaboran un proyecto de intervención y enriquecimiento curricular para ser aplicado en cinco escuelas de las regiones de Cartago, San José y Alajuela. Se realizan algunas actividades previas a su aplicación, prevista para el año académico de 1985; pero, por razones no del todo manifiestas, el proyecto se detiene junto al apoyo económico y a la disposición política para atender, desarrollar y orientar la formación y la creatividad del alumno 
con capacidades altas y talentos especiales. Se busca subsanar este vacío a nivel gubernamental, mediante la creación, en el año 1987, de la sección de "fomento al educando talentoso" adscrita a la Dirección General de Educación Especial (MEP, enero 1992). Tal dependencia se encarga de proponer políticas y acciones específicas para "promover el talento y la creatividad en el sistema educativo costarricense" (MEP, enero 1992, p. 20). Dentro de estas acciones se privilegian la asesoría y capacitación a docentes, la orientación a padres de familia, la investigación, la coordinación entre instituciones y la actualización de datos e información. En el año 1994 este programa se incorpora, como un área de asesoría, a la Sección Escolarizada del mismo Departamento de Educación Especial, con el propósito de detectar al alumno talentoso y promover su desarrollo, en el marco de aula, mediante la orientación a los padres, el apoyo de especialistas y su participación en intervenciones educacionales oportunas y significativas. En 1997, por decisiones políticas, esta Dependencia Asesora se clausura y, de nuevo, se estancan las iniciativas y los intentos estatales de dar una respuesta a las necesidades de este tipo de estudiante.

Este aire de descuido e indiferencia tampoco se renueva a raíz de las tendencias actuales de la Educación Especial que apuestan a una educación integradora e inclusiva, basada en la diferenciación del currículo común, como respuesta educativa a la diversidad. Pues, si bien esta perspectiva educacional permea los documentos oficiales del MEP que norman la atención a los estudiantes con necesidades educativas especiales (NEE), lo hace de manera unilateral y ambigua.

A manera de ejemplo, en el documento de Políticas, Normativa y Procedimientos para el acceso a la Educación de los Estudiantes con NEE (1997), se estipula la siguiente conceptualización:

\footnotetext{
"Alumno con necesidades educativas especiales es
} aquel estudiante que presenta condiciones de aprendizaje diferentes o dificultades en el aprendizaje mayores que el promedio de los alumnos, lo que le dificulta o impide acceder al currículo que le corresponde por su edad, de forma que requiere para compensar dichas diferencias, adecuaciones en una o varias áreas del currículo." (Pág. 5).

Sin lugar a equívocos, el alumno sobresaliente se sitúa en esta conceptualización, dado que es un alumno con "condiciones de aprendizaje diferentes" que lo hacen merecedor de oportunidades y opciones educativas ("adecuaciones en una o varias áreas del currículo") que le permitan un óptimo desarrollo de sus capacidades especiales. Pero, resulta sorprendente como, a partir de esa definición, el documento cierra espacios (ni siquiera conceptualiza el término "condiciones de aprendizaje diferentes") y de una posición inicial de abertura y actitud favorable hacia la diversidad en general, identifica esta última, exclusivamente, con la discapacidad, reduciendo así las necesidades educativas especiales a las dificultades para aprender.

Esta visión reduccionista y controversial con el paradigma actual de Educación Especial, se repite en el documento "Un Centro Educativo Eficiente (MEP, 1998)". Aquí, se define como centro educativo eficaz, aquel que "hace efectivo el derecho a la educación para todas las personas" (pág. 4) a las cuales "abre sus puertas garantizando oportunidades", y se señalan medidas y estrategias aptas para desarrollar una enseñanza eficaz para todos (pp. 5-11). Pero, enseguida, al hacer referencia a las acciones docentes y a la intervención pedagógica (de pág. 12 en adelante), se direcciona la atención, exclusivamente, hacia las dificultades para aprender. En otros términos, de nuevo, en este documento oficial se incurre en la tendencia nefasta de asociar el término "necesidades educativas especiales" con las dificultades de aprendizaje y no con capacidades diversas para aprender.

En fin, dentro de la normativa oficial acerca de la atención a las necesidades educativas especiales no hay referencias 
concretas a los aprendices sobresalientes a sus necesidades y oportunidades de formación. Por el contrario, en ella, si bien se delinea una concepción de Educación Inclusiva y de Educación Especial centrada en los apoyos para optimizar el desarrollo y el aprendizaje de todos los alumnos con necesidades educativas especiales, en el terreno fáctico de las propuestas de intervención pedagógicas, esos alumnos se reducen a aquellos que tienen dificultades y se descuidan los que tienen potencialidades y capacidades superiores $u$ otra diversidad.

A manera conclusiva, observamos, que el educando sobresaliente tiene capacidades elevadas y rasgos peculiares de aprendizaje que lo hacen merecedor, por su derecho a la diversidad, de experiencias educativas diferenciadas que satisfagan sus necesidades educativas especiales y favorezcan el desarrollo pleno de su personalidad. Para este propósito, se cuenta con variadas alternativas educacionales que, dependiendo de la situación contextual de cada caso, se pueden plasmar en el nivel escolar, dentro o fuera de las aulas comunes. Lastimosamente, en el sistema educativo público costarricense, el estudiante con capacidades altas continúa siendo "ignorado", situación que tal vez, se deba a la presencia de prejuicios, y a la idea equívoca y estereotipada, de que él puede hacerlo bien por si mismo, sin contar con herramientas e intervenciones educativas diferenciadas $y$ oportunas. Confiamos en que los principios de igualdad y equidad tan arraigados en la sociedad costarricense induzcan, a quienes compete, a examinar el sistema educativo, y a introducir en él los cambios y las condiciones que hagan realidad en el terreno concreto, el derecho a la diversidad, en su calidad de derecho humano esencial. Solo así, se pueden esperar programas y opciones de formación diversificadas; (no respuestas paliativas $\mathrm{y}$ fugaces), que garanticen a todos los alumnos, y en este caso al alumno capaz y talentoso, el acceso a un aprendizaje significativo. En este camino, esperamos que las ideas aquí plasmadas contribuyan a la sensibilización del lector sobre la problemática educacional que atañe a este colectivo de alumnos y lo induzca a la reflexión crítica y creativa.

\section{Referencias bibliográficas}

Alonso J. A. y Benito Mate J. "Intervención Educativa en niños superdotados". En Benito Mate J. (coord.). Desarrollo y educación de los niños superdotados. Salamanca: Amarú Ediciones, (p. 219-250). 1996.

Beirute L. y De Bravo O. El niño talentoso en Costa Rica: la adecuación de un currículum para el fomento de su creatividad en el aula regular. Costa Rica: Universidad de Costa Rica. 1984.

Benito Mate Y. (coord.). Problemática del niño superdotado. Salamanca: Amarú Ediciones. 2002.

Benito Mate, Y. (coord.). Desarrollo y educación de los niños superdotados. Salamanca: Amarú Ediciones. 1996.

Howell R., Hewards W. y Swassing H. "Los alumnos superdotados." En Herward W. Niños Excepcionales una introducción a la educación especial. Madrid: Prentice Hall, Quinta Edición, (p. 433-481). 1998.

Lafourcade P. y otros. El niño talentoso en Costa Rica. Costa Rica: CEMIE, Instituto Educativo Moderno y MEP. 1982.

Ministerio de Educación Pública. Un centro educativo eficiente. Costa Rica: MEP, Departamento de Educación Especial. 1998. 
Ortiz González M. C. "Hacia una educación inclusiva". La educación especial ayer, hoy y mañana. Salamanca: Siglo Cero, Vol. 31 (1), (p. 5-11). 2000.

Pérez Sánchez, L. (coord.). Diez palabras clave en superdotados. Estella (Navarra): Editorial Verbo Divino. 1993.

Pérez, X. Principios orientadores e información básica de la Asesoría Nacional de Desarrollo y Atención del Talento. Costa Rica: Ministerio de Educación Pública. 1996.

Políticas, Normativa y Procedimientos para el acceso a la educación de los estudiantes con necesidades educativas especiales. Costa Rica: Aprobadas por acuerdo № 18-97, del Consejo Superior de Educación en Sesión del 11 de marzo de 1997. 1997.
Porto, A. M. "Diseño Curricular. Desarrollo y preparación de programas para el estudiante muy capacitado". En: Benito Mate J. Problemática del niño superdotado. Salamanca: Amarú Ediciones, (p. 85-109). 2000.

Roselló C. G. y González J. P. "Intervenciones”. En: Pérez Sánchez L. (coord.). Diez palabras claves en superdotados. Estella (Navarra): Editorial Verbo Divino, (p. 244-273). 1993.

Shea T. M. y Bauer A. M. Educación Especial. Un enfoque ecológico. México: McGraw Hill. 1999.

Stainback S. y W. Aulas inclusivas. Morata, Madrid. 1999.

Torres González J. A. Educación y diversidad. Bases didácticas y organizativas. Málaga: Aljibe. 1999.

Giuseppa D’Agostino Santoro Docente en la Escuela de Orientación y Educación Especial de la Facultad de Educación de la Universidad de Costa Rica 